Qualitative study-other

\section{Greater support and debriefing may help student midwives to process traumatic birth experiences}

$10.1136 /$ eb-2015-102194

\section{Ellen Kitson-Reynolds}

Faculty of Health Sciences, University of Southampton, Southampton, UK

Correspondence to: Dr Ellen Kitson-Reynolds, Faculty of Health Sciences, University of Southampton, Nightingale Building, 67 University Road, Highfield, Southampton, Hants S017 1BJ, UK; elkr@soton.ac.uk

Commentary on: Davies S, Coldridge L. 'No Man's Land': an exploration of the traumatic experiences of student midwives in practice. Midwifery 2015;31:858-64.

\section{Implications for practice and research}

- Incorporating resilience training and a multidisciplinary debriefing approach within clinical and academic environments would develop a culture of support and compassion for the workforce and ultimately effective care for women using maternity services.

- Further research surrounding the effectiveness of resilience education for student midwives is required to elicit greater understanding of the effect on woman-centred care.

\section{Context}

The definition of a midwife is 'being with woman'. Student midwives typically embark on a 156-week education programme developing knowledge and skills predominantly in 'normality' and within the context of normal birth. The reality for many students encompasses exposure to more high-risk complex cases and to services that are resource constrained. Students are immersed into the 'real' world of midwifery care provision from day 1 of their clinical experiences. Davies and Coldridge elicit the experiences of traumatic birth on the emotional impact for student midwives which is commensurate with the growing body of evidence surrounding students ${ }^{1}$ and newly qualified midwives ${ }^{2}$ in this topic area.

\section{Methods}

The purpose of the study was to explore what student midwives' perceived to be traumatic for them and elicit how they were supported with such events. The study is linked to the women's care therefore; the focus is primarily within the clinical context. The study used a qualitative descriptive approach with 11 participants recruited from one university in the North West of England. Participants were recruited from an undisclosed total number of second-year and third-year student midwives by poster campaign displayed on a local website and distributed by midwifery lecturers. Data were gathered through audio-recorded semistructured interviews, field notes, prose and images and analysed using an Interpretive Phenomenological Analysis.

\section{Findings}

Both researchers conducted an iterative process of theme identification from the transcribed interviews and looked for convergence across participants. Five final themes encompassing participants' perceptions of traumatic events include: wearing your blues; no man's land; get the red box; the aftermath; and learning to cope. These indicate that students often have conflicting and competing demands between caring for women, acting as their advocates and completing the requirements of an often busy stressful working environment. These participants identified coping strategies to survive the stressors and the tensions of being a student and 'being with woman'.

\section{Commentary}

Experiences gleaned as student midwives impact on shaping practitioners and the profession of the future. These experiences also determine whether the student will progress to registering with the professional body or decide to leave the profession, ${ }^{2}$ which is akin to seminal research concerning reality shock. ${ }^{3}$ This study focused on conflicts between providing individualised woman-centred care and the realities of working within busy obstetric units where woman-focused philosophies of care appear divergent to the perceived power over women who enter the busy prescriptive clinical environment. Participants articulated their anxiety for when things go wrong and to the levels of changing responsibilities and are reliant on the level of support and role modelling from clinical mentors to alleviate their stress of perceived traumatic events. This study further confirms the findings where being immersed into the realities of clinical practice could ultimately lead to a positive learning experience. ${ }^{4}$

The importance of this study links to supporting a future workforce that is fit for purpose and able to meet the competing demands of the ever changing National Health Service (NHS), while meeting individualised woman-centred care in a cost-effective way. Educationalists are tasked with minimising attrition, through supporting student midwives through challenging events. Hot debriefing ${ }^{5}$ is becoming instrumental in supporting the multiprofessional team following a traumatic and often emergency event. However, this study has highlighted that it is not just emergency situations that can cause stress or trauma for practitioners. Davies and Coldridge highlight the need for supportive cultures to be fostered within clinical arenas and challenges educators to consider incorporating resilience skills to support the student's perceived level of vulnerability. Further detail concerning the data analysis of the prose and images would enhance the study's credibility and transferability, however, findings can be considered for commonality within similar contexts.

\section{Competing interests None declared.}

\section{References}

1. Hughes AJ, Fraser DM. 'Sink or swim': the experience of newly qualified midwives in England. Midwifery 2011;27:382-6.

2. Reynolds EK, Cluett E, Le-May A. Fairy tale midwifery-fact or fiction: the lived experiences of newly qualified midwives. Br J Midwifery 2014;22:660-8.

3. Kramer M. Reality shock: why nurses leave nursing. St. Louis: The C.V Mosby Company, 1974.

4. Fraser DM. Improving learning in hospital labour suites: an ethnographic approach to educational audit. Learn Health Soc Care 2006;5:194-207.

5. Cho SJ. Debriefing in pediatrics. Korean J Pediatr 2015;58:47-51. 